\title{
On Multi-Resolution Coding and a Two-Hop Network
}

\author{
Wei-Hsin Gu and Michelle Effros * \\ whgu@z.caltech.edu and effros@caltech.edu \\ Data Compression Laboratory, California Institute of Technology, Pasadena, CA 91125
}

\begin{abstract}
We study the source coding problem on a simple two-hop network with side information on the middle and end nodes. For the degraded case, where the side information at the end node is weaker than the side information at the middle node, a complete characterization of the rate-distortion region is derived.
\end{abstract}

Consider the network source coding problem shown in the figure. All of the source sequences are drawn i.i.d. according to joint probability mass function $p(x, y, z)$ on finite alphabets $\mathcal{X}, \mathcal{Y}$, and $\mathcal{Z}$. We fix the distortion measure $d$ and define the ratedistortion region as the closure of the set of rate-distortion vectors $\left(R_{1}, R_{2}, D_{X_{1}}, D_{X_{2}}, D_{Y}\right)$ that can be achieved in coding on the given network. Our central result is a complete characterization of this rate-distortion region in the degraded case, when $X \rightarrow Y \rightarrow Z$ forms a Markov chain.

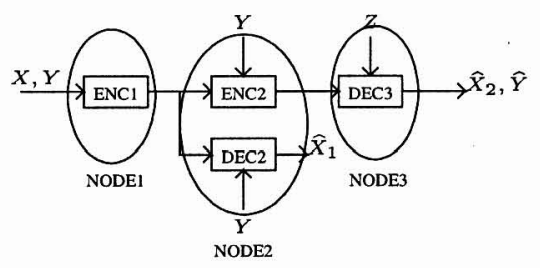

Theorem 1 Suppose $X \rightarrow Y \rightarrow Z$ is a Markov chain. The rate vector $\left(R_{1}, R_{2}\right)$ is in the $\left(D_{X_{1}}, D_{X_{2}}, D_{Y}\right)$-achievable region of the simple two-hop network if and only if there exist finite-alphabet, auxiliary random variables $U$ and $V$ for which $R_{1} \geq$ $I(X ; U, V \mid Y), R_{2} \geq I(X, Y ; V \mid Z)$ and

(i) $(U, V) \rightarrow(X, Y) \rightarrow Z$ forms a Markov chain.

(ii) There exists functions $\widehat{X}_{1}(U, Y), \widehat{X}_{2}(V, Z)$, and $\widehat{Y}(V, Z)$ such that

$$
E\left(d\left(X, \widehat{X}_{1}(U, Y)\right)\right) \leq D_{X_{1}} E(d(X, \widehat{Y}(V, Z))) \leq D_{Y}, E\left(d\left(X, \widehat{X}_{2}(V, Z)\right)\right) \leq D_{X_{2}}
$$

*This material is based upon work partially supported by NSF Grant No. CCR-0220039 and the Caltech's Lee Center for Advanced Networking. 